\title{
Crystal Field Effects on Atomic and Functional-group Distributed Polarizabilities of Molecular Materials
}

\author{
Raphael F. Ligório, ${ }^{a}$ Anna Krawczuk ${ }^{b}$ and Leonardo H. R. Dos Santos ${ }^{a *}$ \\ ${ }^{a}$ Departamento de Química, Universidade Federal de Minas Gerais, Av. Pres. Antônio Carlos 6627, \\ 31270-901 Belo Horizonte MG, Brazil. \\ ${ }^{b}$ Institut für Anorganische Chemie, Unisersität Göttingen, Tammannstrasse 4, D-37077 Göttingen, \\ Germany. \\ * leonardohrs@ufmg.br
}

\section{Supporting Information}

\section{Distributed polarizabilities for all studied molecules}

Table S1: Diagonalized, isotropic and anisotropic polarizabilities for urea calculated using wavefunction methods and correlation-consistent basis sets. Values in a.u.

\begin{tabular}{ccccccc}
\hline & Basis & $\boldsymbol{\alpha}_{\mathbf{1 1}}$ & $\boldsymbol{\alpha}_{\mathbf{2 2}}$ & $\boldsymbol{\alpha}_{33}$ & $\boldsymbol{\alpha}_{\text {iso }}$ & $\Delta \boldsymbol{\alpha}$ \\
\hline HF & cc-pvdz & 13,2 & 27,7 & 30,0 & 23,6 & 15,7 \\
\hline HF & aug-cc-pvdz & 24,1 & 35,1 & 37,1 & 32,1 & 12,1 \\
\hline HF & cc-pvtz & 18,8 & 31,2 & 33,3 & 27,7 & 13,6 \\
\hline HF & aug-cc-pvtz & 24,2 & 35,7 & 37,3 & 32,4 & 12,3 \\
\hline HF & cc-pvqz & 21,4 & 33,3 & 35,1 & 29,9 & 12,9 \\
\hline HF & aug-cc-pvqz & 24,3 & 35,8 & 37,4 & 32,5 & 12,4 \\
\hline MP2 & cc-pvdz & 13,5 & 30,6 & 32,7 & 25,6 & 18,2 \\
\hline MP2 & aug-cc-pvdz & 27,1 & 40,2 & 42,4 & 36,6 & 14,4 \\
\hline MP2 & daug-cc-pvdz & 27,7 & 41,4 & 43,2 & 37,4 & 14,7 \\
\hline MP2 & cc-pvtz & 19,6 & 34,6 & 36,8 & 30,3 & 16,2 \\
\hline MP2 & aug-cc-pvtz & 27,1 & 41,0 & 42,9 & 37,0 & 14,9 \\
\hline MP2 & cc-pvqz & 22,8 & 37,2 & 39,3 & 33,1 & 15,6 \\
\hline MP2 & aug-cc-pvqz & 27,2 & 41,2 & 42,8 & 37,0 & 14,8 \\
\hline CISD & cc-pvdz & 13,3 & 29,0 & 31,2 & 24,5 & 16,9 \\
\hline CISD & aug-cc-pvdz & 25,2 & 37,3 & 39,5 & 34,0 & 13,3 \\
\hline CCSD & cc-pvdz & 13,4 & 30,1 & 32,2 & 25,2 & 17,8 \\
\hline CCSD & aug-cc-pvdz & 26,4 & 39,3 & 41,5 & 35,7 & 14,1 \\
\hline
\end{tabular}


Table S2: Diagonalized, isotropic and anisotropic polarizabilities for urea calculated at the DFT/aug-ccpVDZ level of theory using many functionals. Values in a.u.

\begin{tabular}{|c|c|c|c|c|c|c|}
\hline & Type & $\alpha_{11}$ & $\alpha_{22}$ & $\alpha_{33}$ & $\alpha_{\text {iso }}$ & $\Delta \boldsymbol{\alpha}$ \\
\hline SVWN & LDA & 27,6 & 43,18 & 44,0 & 38,2 & 16,1 \\
\hline BP86 & GGA & 27,7 & 43,5 & 44,2 & 38,5 & 16,2 \\
\hline BLYP & GGA & 28,1 & 44,1 & 44,8 & 39,0 & 16,4 \\
\hline BPW91 & GGA & 27,4 & 43,1 & 43,9 & 38,1 & 16,1 \\
\hline НСТН & GGA & 27,7 & 43,6 & 44,4 & 38,6 & 16,3 \\
\hline OLYP & GGA & 27,9 & 44,1 & 44,6 & 38,8 & 16,5 \\
\hline BB95 & m-GGA & 27,7 & 43,9 & 44,6 & 38,7 & 16,5 \\
\hline VSXC & m-GGA & 27,4 & 42,8 & 43,8 & 38,0 & 15,9 \\
\hline t-HCTH & m-GGA & 27,6 & 43,2 & 44,0 & 38,3 & 16,0 \\
\hline B3P86 & h-GGA & 26,2 & 40,4 & 41,6 & 36,1 & 14,9 \\
\hline B3PW91 & h-GGA & 26,3 & 40,6 & 41,7 & 36,2 & 14,9 \\
\hline B3LYP & h-GGA & 26,7 & 41,1 & 42,3 & 36,7 & 15,1 \\
\hline O3LYP & h-GGA & 27,0 & 42,1 & 42,9 & 37,4 & 15,5 \\
\hline X3LYP & h-GGA & 26,7 & 41,1 & 42,2 & 36,7 & 15,0 \\
\hline BMK & hm-GGA & 24,9 & 38,5 & 39,8 & 34,4 & 14,3 \\
\hline M06-2X & hm-GGA & 25,7 & 39,0 & 40,5 & 35,1 & 14,1 \\
\hline TPSSh & hm-GGA & 26,9 & 41,8 & 42,7 & 37,2 & 15,4 \\
\hline tHCTHhyb & hm-GGA & 26,8 & 41,3 & 42,4 & 36,8 & 15,0 \\
\hline M06 & hm-GGA & 25,6 & 39,9 & 41,0 & 35,5 & 14,9 \\
\hline M06-HF & hm-GGA & 27,2 & 39,0 & 40,8 & 35,7 & 12,8 \\
\hline M05 & hm-GGA & 26,2 & 40,7 & 41,9 & 36,2 & 15,2 \\
\hline M05-2X & hm-GGA & 25,5 & 38,4 & 39,8 & 34,6 & 13,7 \\
\hline CAM-B3LYP & lc-GGA & 26,2 & 39,8 & 41,3 & 35,8 & 14,4 \\
\hline LC-BLYP & lc-GGA & 25,5 & 38,3 & 40,2 & 34,7 & 13,9 \\
\hline wB97 & lc-GGA & 26,0 & 39,0 & 40,8 & 35,3 & 14,0 \\
\hline LC-OLYP & lc-GGA & 25,4 & 38,3 & 40,1 & 34,6 & 13,9 \\
\hline LC-t-HCTH & lc-GGA & 25,6 & 38,4 & 40,3 & 34,8 & 13,8 \\
\hline LC-HCTH & lc-GGA & 25,6 & 38,3 & 40,5 & 34,8 & 14,0 \\
\hline LC-BB95 & lc-GGA & 25,2 & 38,4 & 40,1 & 34,6 & 14,1 \\
\hline LC-BP86 & lc-GGA & 25,3 & 38,3 & 39,9 & 34,5 & 13,9 \\
\hline
\end{tabular}


Table S3: Diagonalized, isotropic and anisotropic polarizabilities for succinic acid calculated using wavefunction methods and correlation-consistent basis sets. Values in a.u.

\begin{tabular}{ccccccc}
\hline & Basis & $\boldsymbol{\alpha}_{\mathbf{1 1}}$ & $\boldsymbol{\alpha}_{\mathbf{2 2}}$ & $\boldsymbol{\alpha}_{33}$ & $\boldsymbol{\alpha}_{\text {iso }}$ & $\Delta \boldsymbol{\alpha}$ \\
\hline HF & cc-pvdz & 33,7 & 51,6 & 59,5 & 48,2 & 22,9 \\
\hline HF & aug-cc-pvdz & 42,9 & 60,8 & 69,8 & 57,8 & 23,7 \\
\hline HF & cc-pvtz & 39,1 & 56,5 & 65,6 & 53,7 & 23,3 \\
\hline HF & aug-cc-pvtz & 43,5 & 61,0 & 70,7 & 58,4 & 23,9 \\
\hline HF & cc-pvqz & 41,0 & 59,2 & 68,6 & 56,3 & 24,3 \\
\hline HF & aug-cc-pvqz & 43,8 & 61,4 & 70,6 & 58,6 & 23,6 \\
\hline MP2 & cc-pvdz & 34,9 & 54,3 & 65,8 & 51,6 & 27,1 \\
\hline MP2 & aug-cc-pvdz & 46,1 & 67,0 & 79,1 & 64,1 & 28,9 \\
\hline MP2 & daug-cc-pvdz & 46,9 & 67,5 & 79,6 & 64,6 & 28,6 \\
\hline MP2 & cc-pvtz & 40,7 & 60,6 & 73,1 & 58,1 & 28,3 \\
\hline MP2 & aug-cc-pvtz & 46,4 & 67,2 & 80,0 & 64,5 & 29,4 \\
\hline MP2 & cc-pvqz & 43,5 & 64,2 & 76,3 & 61,3 & 28,7 \\
\hline MP2 & aug-cc-pvqz & 46,4 & 67,1 & 79,6 & 64,4 & 29,0 \\
\hline CISD & cc-pvdz & 33,9 & 52,3 & 61,6 & 49,3 & 24,4 \\
\hline CISD & aug-cc-pvdz & 43,8 & 62,8 & 72,6 & 59,7 & 25,4 \\
\hline CCSD & cc-pvdz & 34,0 & 53,7 & 64,1 & 50,6 & 26,4 \\
\hline CCSD & aug-cc-pvdz & 45,4 & 65,7 & 75,7 & 62,3 & 26,7 \\
\hline
\end{tabular}


Table S4: Diagonalized, isotropic and anisotropic polarizabilities for succinic acid calculated at the DFT/aug-cc-pVDZ level of theory using many functionals. Values in a.u.

\begin{tabular}{|c|c|c|c|c|c|c|}
\hline & Type & $\alpha_{11}$ & $\alpha_{22}$ & $\alpha_{33}$ & $\boldsymbol{\alpha}_{\text {iso }}$ & $\Delta \boldsymbol{\alpha}$ \\
\hline SVWN & LDA & 47,6 & 69,1 & 87,2 & 68,0 & 34,3 \\
\hline BP86 & GGA & 47,5 & 68,6 & 88,4 & 68,2 & 35,4 \\
\hline BLYP & GGA & 48,0 & 69,6 & 88,4 & 68,7 & 35,1 \\
\hline BPW91 & GGA & 47,1 & 68,2 & 87,5 & 67,6 & 35,0 \\
\hline НСТН & GGA & 47,3 & 67,7 & 87,7 & 67,6 & 35,0 \\
\hline OLYP & GGA & 47,3 & 69,1 & 87,7 & 68,0 & 35,0 \\
\hline BB95 & m-GGA & 47,6 & 69,4 & 88,5 & 68,5 & 35,5 \\
\hline VSXC & m-GGA & 47,4 & 68,7 & 86,0 & 67,4 & 33,5 \\
\hline t-HCTH & m-GGA & 47,2 & 68,7 & 86,6 & 67,5 & 34,2 \\
\hline B3P86 & h-GGA & 45,7 & 66,2 & 81,2 & 64,4 & 30,8 \\
\hline B3PW91 & h-GGA & 45,7 & 66,3 & 81,4 & 64,5 & 31,0 \\
\hline B3LYP & h-GGA & 45,8 & 67,1 & 81,6 & 64,8 & 31,2 \\
\hline O3LYP & h-GGA & 46,4 & 67,5 & 84,0 & 66,0 & 32,7 \\
\hline X3LYP & h-GGA & 45,8 & 66,8 & 81,6 & 64,7 & 31,1 \\
\hline BMK & hm-GGA & 44,2 & 63,7 & 75,5 & 61,1 & 27,4 \\
\hline M06-2X & hm-GGA & 45,2 & 65,2 & 76,9 & 62,4 & 27,7 \\
\hline TPSSh & hm-GGA & 46,3 & 67,3 & 83,6 & 65,8 & 32,4 \\
\hline tHCTHhyb & hm-GGA & 46,4 & 67,1 & 83,0 & 65,5 & 31,8 \\
\hline M06 & hm-GGA & 45,1 & 65,6 & 78,7 & 63,2 & 29,4 \\
\hline M06-HF & hm-GGA & 46,5 & 65,2 & 77,4 & 63,0 & 26,9 \\
\hline M05 & hm-GGA & 45,4 & 66,4 & 81,3 & 64,4 & 31,2 \\
\hline M05-2X & hm-GGA & 44,7 & 64,1 & 76,8 & 61,9 & 28,0 \\
\hline CAM-B3LYP & lc-GGA & 45,9 & 66,0 & 79,3 & 63,7 & 29,1 \\
\hline LC-BLYP & lc-GGA & 45,2 & 65,0 & 75,9 & 62,0 & 27,0 \\
\hline wB97 & lc-GGA & 45,8 & 65,8 & 77,5 & 63,0 & 27,8 \\
\hline LC-OLYP & lc-GGA & 45,0 & 64,8 & 75,8 & 61,8 & 27,0 \\
\hline LC-t-HCTH & lc-GGA & 45,9 & 65,3 & 75,2 & 62,1 & 25,8 \\
\hline LC-HCTH & lc-GGA & 45,5 & 65,2 & 75,9 & 62,2 & 26,7 \\
\hline LC-BB95 & lc-GGA & 44,6 & 64,5 & 75,1 & 61,4 & 26,8 \\
\hline LC-BP86 & lc-GGA & 44,6 & 64,2 & 74,7 & 61,2 & 26,5 \\
\hline
\end{tabular}


Table S5: Diagonalized, isotropic and anisotropic polarizabilities for p-nitroaniline calculated using wavefunction methods and correlation-consistent basis sets. Values in a.u.

\begin{tabular}{ccrrrrr}
\hline & Basis & $\boldsymbol{\alpha}_{\mathbf{1 1}}$ & $\boldsymbol{\alpha}_{\mathbf{2 2}}$ & $\boldsymbol{\alpha}_{\mathbf{3 3}}$ & $\boldsymbol{\alpha}_{\text {iso }}$ & $\Delta \boldsymbol{\alpha}$ \\
\hline HF & cc-pvdz & 31,6 & 89,0 & 123,4 & 81,3 & 80,3 \\
\hline HF & aug-cc-pvdz & 53,7 & 98,2 & 140,1 & 97,3 & 74,8 \\
\hline HF & cc-pvtz & 43,0 & 93,5 & 131,3 & 89,3 & 76,7 \\
\hline HF & aug-cc-pvtz & 53,9 & 98,6 & 140,5 & 97,7 & 75,0 \\
\hline HF & cc-pvqz & 49,2 & 95,8 & 136,1 & 93,7 & 75,4 \\
\hline HF & aug-cc-pvqz & 53,9 & 98,5 & 140,8 & 97,7 & 75,2 \\
\hline MP2 & cc-pvdz & 31,3 & 92,5 & 133,6 & 85,8 & 89,2 \\
\hline MP2 & aug-cc-pvdz & 55,9 & 103,8 & 157,5 & 105,8 & 88,1 \\
\hline MP2 & daug-cc-pvdz & 56,6 & 104,5 & 158,5 & 106,5 & 88,3 \\
\hline MP2 & cc-pvtz & 43,0 & 97,8 & 143,8 & 94,9 & 87,5 \\
\hline MP2 & aug-cc-pvtz & 55,9 & 104,4 & 157,9 & 106,1 & 88,4 \\
\hline MP2 & cc-pvqz & 49,6 & 100,5 & 150,1 & 100,1 & 87,1 \\
\hline MP2 & aug-cc-pvqz & 55,8 & 104,1 & 157,9 & 105,9 & 88,5 \\
\hline CISD & cc-pvdz & 31,3 & 89,7 & 125,8 & 82,3 & 82,6 \\
\hline CISD & aug-cc-pvdz & 53,7 & 99,0 & 144,2 & 99,0 & 78,4 \\
\hline CCSD & cc-pvdz & 31,3 & 90,9 & 127,9 & 83,4 & 84,4 \\
\hline CCSD & aug-cc-pvdz & 55,1 & 101,9 & 150,5 & 102,5 & 82,6 \\
\hline
\end{tabular}


Table S6: Diagonalized, isotropic and anisotropic polarizabilities for p-nitroaniline calculated at the DFT/aug-cc-pVDZ level of theory using many functionals. Values in a.u.

\begin{tabular}{|c|c|c|c|c|c|c|}
\hline & Type & $\alpha_{11}$ & $\alpha_{22}$ & $\alpha_{33}$ & $\boldsymbol{\alpha}_{\text {iso }}$ & $\Delta \boldsymbol{\alpha}$ \\
\hline SVWN & LDA & 55,4 & 103,5 & 171,1 & 110,0 & 100,7 \\
\hline BP86 & GGA & 55,7 & 103,6 & 170,5 & 109,9 & 99,8 \\
\hline BLYP & GGA & 56,0 & 104,4 & 171,8 & 110,7 & 100,8 \\
\hline BPW91 & GGA & 55,2 & 102,8 & 169,5 & 109,2 & 99,5 \\
\hline НСТН & GGA & 54,6 & 103,0 & 170,2 & 109,2 & 100,5 \\
\hline OLYP & GGA & 55,0 & 103,1 & 170,3 & 109,5 & 100,3 \\
\hline BB95 & m-GGA & 55,3 & 103,7 & 171,0 & 110,0 & 100,7 \\
\hline VSXC & m-GGA & 55,4 & 102,9 & 168,1 & 108,8 & 98,0 \\
\hline t-HCТН & m-GGA & 55,6 & 103,4 & 170,4 & 109,8 & 99,9 \\
\hline B3P86 & h-GGA & 54,2 & 100,9 & 161,3 & 105,5 & 93,0 \\
\hline B3PW91 & h-GGA & 54,3 & 100,9 & 161,4 & 105,5 & 93,0 \\
\hline B3LYP & h-GGA & 54,8 & 102,0 & 162,7 & 106,5 & 93,7 \\
\hline O3LYP & h-GGA & 54,4 & 101,9 & 165,3 & 107,2 & 96,4 \\
\hline X3LYP & h-GGA & 54,9 & 102,1 & 162,3 & 106,4 & 93,2 \\
\hline BMK & $\mathrm{hm}-\mathrm{GGA}$ & 53,0 & 99,7 & 154,1 & 102,3 & 87,6 \\
\hline M06-2X & hm-GGA & 53,8 & 100,4 & 152,8 & 102,4 & 85,8 \\
\hline TPSSh & hm-GGA & 54,7 & 101,9 & 164,8 & 107,1 & 95,6 \\
\hline tHCTHhyb & hm-GGA & 55,2 & 102,0 & 164,1 & 107,1 & 94,6 \\
\hline M06 & hm-GGA & 55,6 & 101,5 & 159,9 & 105,6 & 90,6 \\
\hline M06-HF & hm-GGA & 55,8 & 101,2 & 148,2 & 101,7 & 80,0 \\
\hline M05 & hm-GGA & 54,7 & 101,2 & 162,0 & 105,9 & 93,2 \\
\hline M05-2X & hm-GGA & 53,5 & 99,0 & 152,5 & 101,7 & 85,8 \\
\hline CAM-B3LYP & lc-GGA & 54,4 & 101,0 & 155,3 & 103,6 & 87,4 \\
\hline LC-BLYP & lc-GGA & 53,4 & 99,8 & 148,3 & 100,5 & 82,2 \\
\hline wB97 & lc-GGA & 54,6 & 100,7 & 149,4 & 101,6 & 82,1 \\
\hline LC-OLYP & lc-GGA & 53,3 & 99,4 & 148,1 & 100,3 & 82,1 \\
\hline LC-t-HCTH & lc-GGA & 54,4 & 101,4 & 149,0 & 101,6 & 81,9 \\
\hline LC-HCTH & lc-GGA & 53,4 & 100,5 & 148,6 & 100,8 & 82,5 \\
\hline LC-BB95 & lc-GGA & 53,0 & 99,6 & 147,5 & 100,1 & 81,9 \\
\hline LC-BP86 & lc-GGA & 53,4 & 99,5 & 147,4 & 100,1 & 81,4 \\
\hline
\end{tabular}


Table S7: Diagonalized, isotropic and anisotropic polarizabilities for 4-mercaptopyridine calculated using wavefunction methods and correlation-consistent basis sets. Values in a.u.

\begin{tabular}{ccrrrrr}
\hline & Basis & $\boldsymbol{\alpha}_{\mathbf{1 1}}$ & $\boldsymbol{\alpha}_{\mathbf{2 2}}$ & $\boldsymbol{\alpha}_{\mathbf{3 3}}$ & $\boldsymbol{\alpha}_{\text {iso }}$ & $\Delta \boldsymbol{\alpha}$ \\
\hline HF & cc-pvdz & 30,2 & 66,7 & 134,0 & 76,9 & 91,2 \\
\hline HF & aug-cc-pvdz & 56,2 & 83,4 & 147,2 & 95,6 & 80,9 \\
\hline HF & cc-pvtz & 42,1 & 74,5 & 141,5 & 86,0 & 87,8 \\
\hline HF & aug-cc-pvtz & 57,2 & 84,8 & 149,6 & 97,2 & 82,1 \\
\hline HF & cc-pvqz & 49,5 & 79,4 & 145,2 & 91,4 & 84,8 \\
\hline MP2 & cc-pvdz & 29,8 & 70,9 & 142,7 & 81,1 & 98,9 \\
\hline MP2 & aug-cc-pvdz & 57,2 & 89,3 & 162,7 & 103,0 & 93,7 \\
\hline MP2 & daug-cc-pvdz & 59,1 & 91,5 & 163,2 & 104,6 & 92,2 \\
\hline MP2 & cc-pvtz & 41,5 & 78,8 & 151,6 & 90,7 & 97,0 \\
\hline MP2 & aug-cc-pvtz & 57,9 & 90,7 & 162,7 & 103,8 & 92,8 \\
\hline MP2 & cc-pvqz & 49,1 & 83,9 & 156,6 & 96,5 & 95,0 \\
\hline MP2 & aug-cc-pvqz & 57,9 & 90,6 & 162,5 & 103,7 & 92,7 \\
\hline CISD & cc-pvdz & 29,9 & 67,6 & 137,8 & 78,4 & 94,9 \\
\hline CISD & aug-cc-pvdz & 55,7 & 84,5 & 154,0 & 98,1 & 87,5 \\
\hline CCSD & cc-pvdz & 29,8 & 68,4 & 143,1 & 80,4 & 99,7 \\
\hline CCSD & aug-cc-pvdz & 56,6 & 86,5 & 163,5 & 102,2 & 95,6 \\
\hline
\end{tabular}


Table S8: Diagonalized, isotropic and anisotropic polarizabilities for 4-mercaptopyridine calculated at the DFT/aug-cc-pVDZ level of theory using many functionals. Values in a.u.

\begin{tabular}{|c|c|c|c|c|c|c|}
\hline & Type & $\alpha_{11}$ & $\alpha_{22}$ & $\alpha_{33}$ & $\boldsymbol{\alpha}_{\text {iso }}$ & $\Delta \boldsymbol{\alpha}$ \\
\hline SVWN & LDA & 56,6 & 92,2 & 152,5 & 100,4 & 83,9 \\
\hline BP86 & GGA & 57,0 & 92,2 & 153,0 & 100,8 & 84,1 \\
\hline BLYP & GGA & 57,6 & 93,8 & 154,7 & 102,1 & 85,0 \\
\hline BPW91 & GGA & 56,4 & 91,4 & 152,5 & 100,1 & 84,3 \\
\hline НСТН & GGA & 55,8 & 92,2 & 152,6 & 100,2 & 84,6 \\
\hline OLYP & GGA & 56,2 & 92,5 & 153,3 & 100,7 & 84,9 \\
\hline BB95 & m-GGA & 56,6 & 92,7 & 153,2 & 100,8 & 84,5 \\
\hline VSXC & m-GGA & 56,5 & 92,0 & 150,9 & 99,8 & 82,6 \\
\hline t-HCTH & m-GGA & 57,0 & 92,1 & 153,2 & 100,8 & 84,4 \\
\hline B3P86 & h-GGA & 55,6 & 88,5 & 151,1 & 98,4 & 84,0 \\
\hline B3PW91 & h-GGA & 55,8 & 88,8 & 151,3 & 98,6 & 84,0 \\
\hline B3LYP & h-GGA & 56,5 & 89,9 & 153,1 & 99,8 & 85,0 \\
\hline O3LYP & h-GGA & 55,9 & 90,5 & 152,1 & 99,5 & 84,5 \\
\hline X3LYP & h-GGA & 56,7 & 89,9 & 153,4 & 100,0 & 85,1 \\
\hline BMK & hm-GGA & 54,6 & 86,3 & 149,6 & 96,8 & 83,8 \\
\hline M06-2X & hm-GGA & 55,6 & 87,2 & 152,9 & 98,6 & 86,0 \\
\hline TPSSh & hm-GGA & 56,1 & 90,4 & 153,0 & 99,8 & 85,1 \\
\hline tHCTHhyb & hm-GGA & 56,6 & 90,2 & 152,0 & 99,6 & 83,8 \\
\hline M06 & hm-GGA & 57,0 & 89,1 & 152,0 & 99,4 & 83,7 \\
\hline M06-HF & hm-GGA & 58,7 & 87,0 & 160,8 & 102,2 & 91,3 \\
\hline M05 & hm-GGA & 56,1 & 89,8 & 153,1 & 99,7 & 85,3 \\
\hline M05-2X & hm-GGA & 55,5 & 86,1 & 151,4 & 97,7 & 84,9 \\
\hline CAM-B3LYP & lc-GGA & 56,0 & 87,5 & 153,4 & 99,0 & 86,0 \\
\hline LC-BLYP & lc-GGA & 54,8 & 84,8 & 153,4 & 97,7 & 87,5 \\
\hline wB97 & lc-GGA & 55,5 & 85,4 & 152,6 & 97,8 & 86,1 \\
\hline LC-OLYP & 1c-GGA & 54,6 & 84,6 & 152,6 & 97,2 & 86,9 \\
\hline LC-t-HCTH & lc-GGA & 56,2 & 85,8 & 155,0 & 99,0 & 87,7 \\
\hline LC-HCTH & 1c-GGA & 55,1 & 85,5 & 153,9 & 98,2 & 87,7 \\
\hline LC-BB95 & lc-GGA & 54,1 & 84,5 & 152,4 & 97,0 & 87,2 \\
\hline LC-BP86 & lc-GGA & 54,5 & 84,4 & 152,0 & 97,0 & 86,5 \\
\hline
\end{tabular}


Table S9: Diagonalized, isotropic and anisotropic polarizabilities for methylbenzoate calculated using wavefunction methods and correlation-consistent basis sets. Values in a.u.

\begin{tabular}{ccrrrrr}
\hline & Basis & $\boldsymbol{\alpha}_{\mathbf{1 1}}$ & $\boldsymbol{\alpha}_{\mathbf{2 2}}$ & $\boldsymbol{\alpha}_{\mathbf{3 3}}$ & $\boldsymbol{\alpha}_{\text {iso }}$ & $\Delta \boldsymbol{\alpha}$ \\
\hline HF & cc-pvdz & 40,0 & 90,5 & 114,4 & 81,6 & 65,9 \\
\hline HF & aug-cc-pvdz & 60,7 & 99,9 & 126,8 & 95,8 & 57,6 \\
\hline HF & cc-pvtz & 51,0 & 95,6 & 121,4 & 89,3 & 61,7 \\
\hline HF & aug-cc-pvtz & 60,7 & 100,1 & 127,0 & 95,9 & 57,7 \\
\hline HF & aug-cc-pvqz & 60,7 & 100,1 & 126,9 & 95,9 & 57,7 \\
\hline MP2 & cc-pvdz & 39,8 & 92,6 & 120,1 & 84,2 & 70,6 \\
\hline MP2 & aug-cc-pvdz & 62,3 & 104,9 & 135,9 & 101,0 & 64,0 \\
\hline MP2 & daug-cc-pvdz & 62,7 & 105,2 & 135,5 & 101,1 & 63,3 \\
\hline MP2 & cc-pvtz & 51,1 & 98,8 & 129,2 & 93,1 & 68,1 \\
\hline MP2 & aug-cc-pvtz & 62,1 & 105,0 & 136,2 & 101,1 & 64,5 \\
\hline MP2 & cc-pvqz & 57,1 & 101,7 & 132,9 & 97,2 & 66,0 \\
\hline MP2 & aug-cc-pvqz & 61,9 & 104,6 & 136,0 & 100,8 & 64,5 \\
\hline CISD & cc-pvdz & 39,6 & 90,7 & 115,4 & 81,9 & 66,9 \\
\hline CISD & aug-cc-pvdz & 60,4 & 100,5 & 127,9 & 96,2 & 58,8 \\
\hline CCSD & cc-pvdz & 39,7 & 91,4 & 116,3 & 82,5 & 67,7 \\
\hline CCSD & aug-cc-pvdz & 61,3 & 102,6 & 130,9 & 98,3 & 60,6 \\
\hline
\end{tabular}


Table S10: Diagonalized, isotropic and anisotropic polarizabilities for methylbenzoate calculated at the DFT/aug-cc-pVDZ level of theory using many functionals. Values in a.u.

\begin{tabular}{|c|c|c|c|c|c|c|}
\hline & Type & $\alpha_{11}$ & $\alpha_{22}$ & $\alpha_{33}$ & $\boldsymbol{\alpha}_{\text {iso }}$ & $\Delta \boldsymbol{\alpha}$ \\
\hline SVWN & LDA & 62,6 & 107,5 & 146,3 & 105,5 & 72,5 \\
\hline BP86 & GGA & 62,9 & 107,4 & 146,5 & 105,6 & 72,5 \\
\hline BLYP & GGA & 63,1 & 108,0 & 146,8 & 106,0 & 72,5 \\
\hline BPW91 & GGA & 62,1 & 106,6 & 145,4 & 104,7 & 72,1 \\
\hline НСТН & GGA & 61,5 & 106,9 & 145,0 & 104,5 & 72,4 \\
\hline OLYP & GGA & 61,7 & 106,8 & 145,3 & 104,6 & 72,5 \\
\hline BB95 & m-GGA & 62,3 & 107,6 & 146,8 & 105,6 & 73,2 \\
\hline VSXC & m-GGA & 62,3 & 106,3 & 143,8 & 104,1 & 70,6 \\
\hline t-HCTH & m-GGA & 62,5 & 106,9 & 145,3 & 104,9 & 71,8 \\
\hline B3P86 & h-GGA & 61,2 & 104,3 & 139,8 & 101,8 & 68,1 \\
\hline B3PW91 & h-GGA & 61,2 & 104,4 & 139,5 & 101,7 & 67,9 \\
\hline B3LYP & h-GGA & 61,8 & 105,3 & 140,5 & 102,5 & 68,2 \\
\hline O3LYP & h-GGA & 61,2 & 105,3 & 141,5 & 102,6 & 69,7 \\
\hline X3LYP & h-GGA & 61,9 & 105,4 & 140,2 & 102,5 & 68,0 \\
\hline BMK & hm-GGA & 60,3 & 102,8 & 135,1 & 99,4 & 65,0 \\
\hline M06-2X & hm-GGA & 60,7 & 103,3 & 134,9 & 99,6 & 64,4 \\
\hline TPSSh & hm-GGA & 61,6 & 105,2 & 141,7 & 102,8 & 69,5 \\
\hline tHCTHhyb & hm-GGA & 62,1 & 105,5 & 141,6 & 103,1 & 68,9 \\
\hline M06 & hm-GGA & 62,9 & 104,7 & 138,9 & 102,2 & 66,0 \\
\hline M06-HF & hm-GGA & 61,4 & 102,2 & 131,4 & 98,3 & 60,8 \\
\hline M05 & hm-GGA & 61,5 & 104,3 & 138,1 & 101,3 & 66,5 \\
\hline M05-2X & hm-GGA & 60,2 & 101,8 & 133,7 & 98,6 & 63,8 \\
\hline CAM-B3LYP & lc-GGA & 61,4 & 104,2 & 136,1 & 100,6 & 65,0 \\
\hline LC-BLYP & lc-GGA & 60,3 & 102,3 & 131,2 & 97,9 & 61,8 \\
\hline wB97 & lc-GGA & 61,5 & 103,3 & 131,3 & 98,7 & 60,8 \\
\hline LC-OLYP & lc-GGA & 60,1 & 102,0 & 131,0 & 97,7 & 61,7 \\
\hline LC-t-HCTH & lc-GGA & 62,0 & 104,2 & 133,1 & 99,8 & 61,9 \\
\hline LC-HCTH & lc-GGA & 60,5 & 103,1 & 131,2 & 98,3 & 61,7 \\
\hline LC-BB95 & lc-GGA & 59,8 & 102,0 & 131,0 & 97,6 & 62,0 \\
\hline LC-BP86 & lc-GGA & 60,3 & 101,9 & 130,9 & 97,7 & 61,5 \\
\hline
\end{tabular}




\section{Distributed atomic polarizabilities for all molecules}

Table S11: Diagonalized, isotropic and anisotropic polarizabilities for urea calculated at the M06-HF/augcc-pVDZ level of theory. Values in a.u.

\begin{tabular}{cccccc}
\hline ATOM & $\boldsymbol{\alpha}_{\mathbf{1 1}}$ & $\boldsymbol{\alpha}_{\mathbf{2 2}}$ & $\boldsymbol{\alpha}_{33}$ & $\boldsymbol{\alpha}_{\text {iso }}$ & $\Delta \boldsymbol{\alpha}$ \\
\hline C1 & 1,5 & 4,3 & 4,6 & 3,5 & 2,9 \\
\hline O2 & 6,1 & 6,5 & 13,7 & 8,7 & 7,4 \\
\hline N3 & 6,5 & 8,8 & 13,9 & 9,7 & 6,6 \\
\hline H4 & 0,4 & 0,4 & 2,1 & 0,9 & 1,7 \\
\hline H5 & 0,4 & 0,4 & 2,3 & 1,1 & 1,9 \\
\hline N6 & 6,5 & 8,8 & 13,9 & 9,7 & 6,6 \\
\hline H7 & 0,4 & 0,4 & 2,1 & 0,9 & 1,7 \\
\hline H8 & 0,4 & 0,4 & 2,3 & 1,1 & 1,9 \\
\hline
\end{tabular}

Table S12: Diagonalized, isotropic and anisotropic polarizabilities for succinic acid calculated at the M06-HF/aug-cc-pVDZ level of theory. Values in a.u.

\begin{tabular}{cccccc}
\hline $\mathbf{A T O M}$ & $\boldsymbol{\alpha 1 1}_{11}$ & $\boldsymbol{\alpha 2 2}$ & $\boldsymbol{\alpha 3 3}$ & $\boldsymbol{\alpha}$ iso & $\Delta \boldsymbol{\alpha}$ \\
\hline $\mathbf{O 1}$ & 5,6 & 5,8 & 11,4 & 7,6 & 5,7 \\
\hline $\mathbf{O 2}$ & 5,2 & 5,4 & 12,0 & 7,5 & 6,8 \\
\hline $\mathbf{C 3}$ & 2,2 & 3,9 & 8,0 & 4,7 & 5,1 \\
\hline H4 & 4,3 & 5,4 & 9,8 & 6,5 & 5,1 \\
\hline H6 & 0,2 & 0,3 & 2,0 & 0,9 & 1,8 \\
\hline H7 & 1,1 & 1,4 & 4,0 & 2,2 & 2,8 \\
\hline C8 & 4,4 & 1,3 & 3,8 & 2,1 & 2,6 \\
\hline C9 & 1,9 & 5,5 & 9,9 & 6,6 & 5,1 \\
\hline O10 & 5,7 & 5,8 & 11,4 & 7,6 & 5,7 \\
\hline O11 & 5,2 & 5,3 & 11,9 & 7,5 & 6,7 \\
\hline H12 & 0,2 & 0,3 & 2,0 & 0,9 & 1,8 \\
\hline H13 & 1,1 & 1,4 & 4,0 & 2,2 & 2,8 \\
\hline H14 & 1,1 & 1,3 & 3,8 & 2,1 & 2,6 \\
\hline
\end{tabular}


Table S13: Diagonalized, isotropic and anisotropic polarizabilities for p-nitroaniline calculated at the M06-HF/aug-cc-pVDZ level of theory. Values in a.u.

\begin{tabular}{cccccc}
\hline $\mathbf{A T O M}$ & $\boldsymbol{\alpha}_{\mathbf{1 1}}$ & $\boldsymbol{\alpha}_{\mathbf{2 2}}$ & $\boldsymbol{\alpha}_{33}$ & $\boldsymbol{\alpha}_{\text {iso }}$ & $\Delta \boldsymbol{\alpha}$ \\
\hline $\mathbf{C 1}$ & 4,8 & 10,2 & 14,1 & 9,7 & 8,1 \\
\hline $\mathbf{N 2}$ & 2,4 & 8,0 & 19,5 & 9,9 & 15,1 \\
\hline $\mathbf{C 3}$ & 5,0 & 6,9 & 14,0 & 8,6 & 8,2 \\
\hline $\mathbf{H 4}$ & 0,8 & 1,0 & 3,7 & 1,9 & 2,8 \\
\hline $\mathbf{C 5}$ & 4,9 & 6,7 & 14,0 & 8,5 & 8,3 \\
\hline $\mathbf{H 6}$ & 0,8 & 1,0 & 3,7 & 1,8 & 2,8 \\
\hline $\mathbf{C 7}$ & 6,2 & 7,9 & 15,1 & 9,7 & 8,2 \\
\hline $\mathbf{H 8}$ & 1,0 & 1,2 & 5,0 & 2,4 & 3,9 \\
\hline $\mathbf{C 9}$ & 3,3 & 9,4 & 12,1 & 8,3 & 7,8 \\
\hline $\mathbf{C 1 0}$ & 6,1 & 7,8 & 15,0 & 9,6 & 8,2 \\
\hline $\mathbf{H 1 1}$ & 1,0 & 1,2 & 5,0 & 2,4 & 3,9 \\
\hline $\mathbf{N 1 2}$ & 5,6 & 8,5 & 21,9 & 12,0 & 15,0 \\
\hline $\mathbf{H 1 3}$ & 0,3 & 0,4 & 2,6 & 1,1 & 2,2 \\
\hline $\mathbf{H 1 4}$ & 0,3 & 0,4 & 2,6 & 1,1 & 2,2 \\
\hline $\mathbf{O 1 5}$ & 4,2 & 4,7 & 13,1 & 7,3 & 8,6 \\
\hline $\mathbf{O 1 6}$ & 4,2 & 4,6 & 13,0 & 7,3 & 8,6 \\
\hline
\end{tabular}

Table S14: Diagonalized, isotropic and anisotropic polarizabilities for 4-mercaptopyridine calculated at the M06-HF/aug-cc-pVDZ level of theory. Values in a.u.

\begin{tabular}{cccccc}
\hline $\mathbf{A T O M}$ & $\boldsymbol{\alpha}_{\mathbf{1 1}}$ & $\boldsymbol{\alpha}_{\mathbf{2 2}}$ & $\boldsymbol{\alpha}_{\mathbf{3 3}}$ & $\boldsymbol{\alpha}_{\text {iso }}$ & $\boldsymbol{\Delta} \boldsymbol{\alpha}$ \\
\hline $\mathbf{S 1}$ & 21,2 & 21,9 & 38,7 & 27,3 & 17,2 \\
\hline $\mathbf{N 2}$ & 5,7 & 13,9 & 14,0 & 11,2 & 8,3 \\
\hline $\mathbf{H 3}$ & 0,4 & 0,4 & 2,9 & 1,3 & 2,5 \\
\hline $\mathbf{C 4}$ & 4,9 & 6,9 & 13,0 & 8,3 & 7,3 \\
\hline $\mathbf{H 5}$ & 0,9 & 1,1 & 5,0 & 2,4 & 4,0 \\
\hline $\mathbf{C 6}$ & 4,9 & 6,9 & 13,1 & 8,3 & 7,4 \\
\hline $\mathbf{H 7}$ & 0,9 & 1,1 & 5,0 & 2,4 & 4,0 \\
\hline $\mathbf{C 8}$ & 5,6 & 6,0 & 18,0 & 9,9 & 12,2 \\
\hline $\mathbf{H 9}$ & 0,7 & 1,1 & 4,1 & 1,9 & 3,2 \\
\hline $\mathbf{C 1 0}$ & 5,5 & 5,9 & 18,0 & 9,8 & 12,3 \\
\hline $\mathbf{H 1 1}$ & 0,7 & 1,1 & 4,1 & 1,9 & 3,2 \\
\hline $\mathbf{C 1 2}$ & 5,4 & 8,8 & 38,7 & 17,6 & 31,8 \\
\hline
\end{tabular}


Table S15: Diagonalized, isotropic and anisotropic polarizabilities for methylbenzoate calculated at the M06-HF/aug-cc-pVDZ level of theory. Values in a.u.

\begin{tabular}{cccccc}
\hline ATOM & $\boldsymbol{\alpha}_{11}$ & $\boldsymbol{\alpha}_{22}$ & $\boldsymbol{\alpha}_{33}$ & $\boldsymbol{\alpha}_{\text {iso }}$ & $\Delta \boldsymbol{\alpha}$ \\
\hline $\mathbf{C 1}$ & 4,7 & 10,1 & 15,1 & 10,0 & 9,0 \\
\hline $\mathbf{C 2}$ & 5,2 & 7,4 & 12,6 & 8,4 & 6,6 \\
\hline $\mathbf{H 3}$ & 1,0 & 1,0 & 4,0 & 2,0 & 3,0 \\
\hline $\mathbf{C 4}$ & 5,7 & 8,3 & 11,9 & 8,6 & 5,4 \\
\hline H5 & 1,1 & 1,3 & 5,3 & 2,6 & 4,1 \\
\hline C6 & 5,8 & 9,9 & 10,1 & 8,6 & 4,2 \\
\hline H7 & 1,1 & 1,2 & 5,5 & 2,6 & 4,3 \\
\hline C8 & 5,9 & 8,8 & 11,7 & 8,8 & 5,1 \\
\hline H9 & 1,1 & 1,2 & 5,3 & 2,5 & 4,1 \\
\hline C10 & 5,1 & 7,1 & 12,7 & 8,3 & 6,8 \\
\hline H11 & 1,0 & 1,1 & 4,0 & 2,0 & 3,0 \\
\hline C12 & 2,1 & 4,0 & 11,8 & 5,9 & 8,9 \\
\hline C13 & 3,9 & 4,6 & 6,2 & 4,9 & 2,0 \\
\hline H14 & 1,3 & 1,3 & 4,7 & 2,4 & 3,4 \\
\hline H15 & 1,1 & 1,4 & 4,2 & 2,2 & 2,9 \\
\hline H16 & 1,1 & 1,4 & 4,1 & 2,2 & 2,9 \\
\hline O17 & 5,1 & 5,5 & 12,4 & 7,6 & 7,1 \\
\hline O18 & 3,8 & 5,0 & 17,0 & 8,6 & 12,6 \\
\hline
\end{tabular}

\section{Distributed polarizabilities for functional groups}

Table S16: Diagonalized, isotropic and anisotropic polarizabilities for $-\mathrm{NO}_{2}$ calculated using wavefunction methods and correlation-consistent basis sets, and M06-HF/aug-cc-pVDZ. Values in a.u.

\begin{tabular}{cccccccc}
\hline & Basis & $\boldsymbol{\alpha} 11$ & $\boldsymbol{\alpha 2 2}$ & $\boldsymbol{\alpha 3 3}$ & $\boldsymbol{\alpha}$ iso & $\Delta \boldsymbol{\alpha}$ & $\Delta \boldsymbol{\alpha} / \boldsymbol{\alpha}_{\text {iso }}$ \\
\hline HF & cc-pvdz & 5,4 & 21,0 & 26,8 & 17,7 & 19,2 & 1,08 \\
\hline HF & cc-pvtz & 7,8 & 22,6 & 29,8 & 20,1 & 19,4 & 0,97 \\
\hline HF & cc-pvqz & 9,4 & 23,5 & 32,0 & 21,6 & 19,8 & 0,92 \\
\hline HF & aug-cc-pvdz & 10,6 & 24,1 & 33,8 & 22,8 & 20,2 & 0,89 \\
\hline HF & aug-cc-pvtz & 10,9 & 24,4 & 33,8 & 23,0 & 20,0 & 0,87 \\
\hline HF & aug-cc-pvqz & 10,9 & 24,4 & 33,9 & 23,1 & 20,0 & 0,87 \\
\hline MP2 & cc-pvdz & 5,6 & 21,0 & 29,3 & 18,6 & 20,8 & 1,12 \\
\hline MP2 & cc-pvtz & 7,9 & 23,0 & 33,2 & 21,4 & 22,0 & 1,03 \\
\hline MP2 & cc-pvqz & 9,7 & 24,3 & 36,0 & 23,3 & 22,9 & 0,98 \\
\hline MP2 & aug-cc-pvdz & 11,3 & 25,6 & 39,1 & 25,3 & 24,1 & 0,95 \\
\hline MP2 & daug-cc-pvdz & 11,9 & 26,0 & 39,4 & 25,8 & 23,9 & 0,93 \\
\hline MP2 & aug-cc-pvtz & 11,7 & 25,8 & 39,0 & 25,5 & 23,7 & 0,93 \\
\hline MP2 & aug-cc-pvqz & 11,7 & 25,7 & 39,2 & 25,5 & 23,8 & 0,93 \\
\hline CISD & cc-pvdz & 5,5 & 21,1 & 27,7 & 18,1 & 19,8 & 1,09 \\
\hline CISD & aug-cc-pvdz & 10,7 & 24,7 & 35,3 & 23,6 & 21,4 & 0,91 \\
\hline CCSD & cc-pvdz & 5,6 & 21,6 & 28,0 & 18,4 & 20,0 & 1,09 \\
\hline CCSD & aug-cc-pvdz & 11,1 & 25,9 & 37,2 & 24,7 & 22,6 & 0,91 \\
\hline M06HF & aug-cc-pvdz & 11,7 & 25,9 & 35,9 & 24,5 & 21,1 & 0,86 \\
\hline
\end{tabular}


Table S17: Diagonalized, isotropic and anisotropic polarizabilities for $-\mathrm{NH}_{2}$ calculated using wavefunction methods and correlation-consistent basis sets, and M06-HF/aug-cc-pVDZ. Values in a.u.

\begin{tabular}{cccccccc}
\hline & Basis & $\boldsymbol{\alpha}_{\mathbf{1 1}}$ & $\boldsymbol{\alpha}_{\mathbf{2 2}}$ & $\boldsymbol{\alpha}_{\mathbf{3 3}}$ & $\boldsymbol{\alpha}_{\text {iso }}$ & $\Delta \boldsymbol{\alpha}$ & $\Delta \boldsymbol{\alpha} / \boldsymbol{\alpha}_{\text {iso }}$ \\
\hline HF & cc-pvdz & 4,3 & 6,6 & 19,2 & 10,1 & 13,9 & 1,38 \\
\hline HF & cc-pvtz & 6,4 & 7,4 & 20,4 & 11,4 & 13,6 & 1,19 \\
\hline HF & cc-pvqz & 7,2 & 7,8 & 21,3 & 12,1 & 13,8 & 1,14 \\
\hline HF & aug-cc-pvdz & 8,1 & 8,1 & 22,4 & 12,9 & 14,3 & 1,11 \\
\hline HF & aug-cc-pvtz & 8,1 & 8,2 & 22,4 & 12,9 & 14,3 & 1,11 \\
\hline HF & aug-cc-pvqz & 8,1 & 8,2 & 22,5 & 12,9 & 14,4 & 1,12 \\
\hline MP2 & cc-pvdz & 4,3 & 6,7 & 21,5 & 10,8 & 16,1 & 1,49 \\
\hline MP2 & cc-pvtz & 6,6 & 7,5 & 22,9 & 12,3 & 15,9 & 1,29 \\
\hline MP2 & cc-pvqz & 7,8 & 7,9 & 24,2 & 13,3 & 16,4 & 1,23 \\
\hline MP2 & aug-cc-pvdz & 8,5 & 9,3 & 26,1 & 14,6 & 17,2 & 1,18 \\
\hline MP2 & daug-cc-pvdz & 8,6 & 9,4 & 26,5 & 14,8 & 17,5 & 1,18 \\
\hline MP2 & aug-cc-pvtz & 8,6 & 9,2 & 26,1 & 14,6 & 17,2 & 1,18 \\
\hline MP2 & aug-cc-pvqz & 8,5 & 9,2 & 26,2 & 14,7 & 17,4 & 1,18 \\
\hline CISD & cc-pvdz & 4,3 & 6,6 & 19,9 & 10,3 & 14,6 & 1,42 \\
\hline CISD & aug-cc-pvdz & 8,2 & 8,4 & 23,5 & 13,4 & 15,2 & 1,13 \\
\hline CCSD & cc-pvdz & 4,3 & 6,6 & 20,8 & 10,6 & 15,5 & 1,46 \\
\hline CCSD & aug-cc-pvdz & 8,5 & 9,0 & 25,2 & 14,2 & 16,5 & 1,16 \\
\hline M06HF & aug-cc-pvdz & 8,5 & 9,4 & 24,9 & 14,2 & 15,9 & 1,12 \\
\hline
\end{tabular}

Table S18: Diagonalized, isotropic and anisotropic polarizabilities for -S calculated using wavefunction methods and correlation-consistent basis sets, and M06-HF/aug-cc-pVDZ. Values in a.u.

\begin{tabular}{cccccccc}
\hline & Basis & $\boldsymbol{\alpha} 11$ & $\boldsymbol{\alpha 2 2}_{22}$ & $\boldsymbol{\alpha} 33$ & $\boldsymbol{\alpha}$ iso & $\Delta \boldsymbol{\alpha}$ & $\Delta \boldsymbol{\alpha} / \boldsymbol{\alpha}_{\text {iso }}$ \\
\hline HF & cc-pvdz & 7,3 & 8,5 & 27,8 & 14,6 & 20,0 & 1,37 \\
\hline HF & cc-pvtz & 11,8 & 13,6 & 31,8 & 19,1 & 19,1 & 1,00 \\
\hline HF & cc-pvqz & 15,9 & 17,2 & 33,9 & 22,4 & 17,4 & 0,78 \\
\hline HF & aug-cc-pvdz & 20,0 & 20,2 & 35,3 & 25,2 & 15,2 & 0,60 \\
\hline HF & aug-cc-pvtz & 21,3 & 21,4 & 35,9 & 26,2 & 14,5 & 0,55 \\
\hline HF & aug-cc-pvqz & 21,6 & 21,7 & 36,1 & 26,4 & 14,4 & 0,55 \\
\hline MP2 & cc-pvdz & 7,3 & 8,3 & 30,5 & 15,4 & 22,7 & 1,47 \\
\hline MP2 & cc-pvtz & 11,7 & 13,3 & 36,8 & 20,6 & 24,3 & 1,18 \\
\hline MP2 & cc-pvqz & 15,6 & 17,1 & 39,3 & 24,0 & 23,0 & 0,96 \\
\hline MP2 & aug-cc-pvdz & 20,1 & 20,6 & 39,4 & 26,7 & 19,0 & 0,71 \\
\hline MP2 & daug-cc-pvdz & 21,8 & 22,4 & 39,4 & 27,9 & 17,4 & 0,62 \\
\hline MP2 & aug-cc-pvtz & 21,6 & 22,0 & 42,1 & 28,5 & 20,4 & 0,72 \\
\hline MP2 & aug-cc-pvqz & 21,7 & 22,2 & 42,1 & 28,7 & 20,1 & 0,70 \\
\hline CISD & cc-pvdz & 7,3 & 8,4 & 28,9 & 14,9 & 21,1 & 1,42 \\
\hline CISD & aug-cc-pvdz & 19,9 & 20,0 & 36,6 & 25,5 & 16,7 & 0,65 \\
\hline CCSD & cc-pvdz & 7,3 & 8,4 & 30,0 & 15,2 & 22,2 & 1,46 \\
\hline CCSD & aug-cc-pvdz & 19,9 & 20,6 & 38,4 & 26,3 & 18,2 & 0,69 \\
\hline M06HF & aug-cc-pvdz & 21,2 & 21,9 & 38,7 & 27,3 & 17,2 & 0,63 \\
\hline
\end{tabular}


Table S19: Diagonalized, isotropic and anisotropic polarizabilities for - $\mathrm{COOH}$ calculated using wavefunction methods and correlation-consistent basis sets, and M06-HF/aug-cc-pVDZ. Values in a.u.

\begin{tabular}{cccccccc}
\hline & Basis & $\boldsymbol{\alpha}_{\mathbf{1 1}}$ & $\boldsymbol{\alpha}_{\mathbf{2 2}}$ & $\boldsymbol{\alpha}_{\mathbf{3 3}}$ & $\boldsymbol{\alpha}_{\text {iso }}$ & $\Delta \boldsymbol{\alpha}$ & $\Delta \boldsymbol{\alpha} / \boldsymbol{\alpha}_{\text {iso }}$ \\
\hline HF & cc-pvdz & 7,6 & 16,5 & 19,9 & 14,7 & 11,1 & 0,76 \\
\hline HF & cc-pvtz & 10,0 & 18,7 & 22,3 & 17,0 & 10,9 & 0,64 \\
\hline HF & cc-pvqz & 11,2 & 19,8 & 23,6 & 18,2 & 11,0 & 0,60 \\
\hline HF & aug-cc-pvdz & 11,9 & 20,6 & 24,2 & 18,9 & 10,9 & 0,58 \\
\hline HF & aug-cc-pvtz & 12,1 & 20,6 & 24,6 & 19,1 & 11,0 & 0,58 \\
\hline MP2 & aug-cc-pvqz & 12,2 & 20,6 & 24,4 & 19,1 & 10,9 & 0,57 \\
\hline MP2 & cc-pvtz & 10,5 & 20,4 & 24,3 & 18,4 & 12,3 & 0,67 \\
\hline MP2 & cc-pvqz & 11,9 & 22,0 & 25,7 & 19,9 & 12,3 & 0,62 \\
\hline MP2 & aug-cc-pvdz & 13,1 & 23,4 & 26,9 & 21,1 & 12,4 & 0,59 \\
\hline MP2 & daug-cc-pvdz & 13,5 & 23,6 & 27,0 & 21,4 & 12,2 & 0,57 \\
\hline MP2 & aug-cc-pvtz & 13,3 & 23,5 & 27,2 & 21,3 & 12,4 & 0,58 \\
\hline MP2 & aug-cc-pvqz & 13,4 & 23,4 & 27,1 & 21,3 & 12,3 & 0,58 \\
\hline CISD & cc-pvdz & 7,6 & 17,0 & 20,5 & 15,0 & 11,5 & 0,77 \\
\hline CISD & aug-cc-pvdz & 12,2 & 21,6 & 24,8 & 19,5 & 11,3 & 0,58 \\
\hline CCSD & cc-pvdz & 7,8 & 17,7 & 21,3 & 15,6 & 12,2 & 0,78 \\
\hline CCSD & aug-cc-pvdz & 12,9 & 22,7 & 25,9 & 20,5 & 11,7 & 0,57 \\
\hline M06HF & aug-cc-pvdz & 13,5 & 22,3 & 26,2 & 20,7 & 11,3 & 0,55 \\
\hline
\end{tabular}

Table S20: Diagonalized, isotropic and anisotropic polarizabilities for $-\mathrm{COO}^{-}$calculated using wavefunction methods and correlation-consistent basis sets, and M06-HF/aug-cc-pVDZ. Values in a.u.

\begin{tabular}{cccccccc}
\hline & Basis & $\boldsymbol{\alpha} 11$ & $\boldsymbol{\alpha} 22$ & $\boldsymbol{\alpha 3 3}$ & $\boldsymbol{\alpha}$ iso & $\Delta \boldsymbol{\alpha}$ & $\Delta \boldsymbol{\alpha} / \boldsymbol{\alpha}_{\text {iso }}$ \\
\hline HF & cc-pvdz & 6,9 & 15,4 & 28,7 & 17,0 & 19,0 & 1,12 \\
\hline HF & cc-pvtz & 8,9 & 17,3 & 31,0 & 19,1 & 19,3 & 1,01 \\
\hline HF & cc-pvqz & 9,8 & 18,3 & 31,9 & 20,0 & 19,2 & 0,96 \\
\hline HF & aug-cc-pvdz & 10,3 & 18,9 & 32,3 & 20,5 & 19,3 & 0,94 \\
\hline HF & aug-cc-pvtz & 10,5 & 18,9 & 32,7 & 20,7 & 19,4 & 0,94 \\
\hline HF & aug-cc-pvqz & 10,5 & 18,9 & 32,5 & 20,6 & 19,2 & 0,93 \\
\hline MP2 & cc-pvdz & 7,1 & 17,0 & 30,9 & 18,3 & 20,7 & 1,13 \\
\hline MP2 & cc-pvtz & 9,3 & 19,4 & 33,7 & 20,8 & 21,2 & 1,02 \\
\hline MP2 & cc-pvqz & 10,5 & 20,7 & 35,2 & 22,1 & 21,5 & 0,97 \\
\hline MP2 & aug-cc-pvdz & 11,3 & 21,8 & 35,6 & 22,9 & 21,1 & 0,92 \\
\hline MP2 & daug-cc-pvdz & 11,6 & 21,9 & 35,3 & 23,0 & 20,7 & 0,90 \\
\hline MP2 & aug-cc-pvtz & 11,5 & 21,9 & 35,8 & 23,0 & 21,2 & 0,92 \\
\hline MP2 & aug-cc-pvqz & 11,5 & 21,8 & 36,0 & 23,1 & 21,3 & 0,92 \\
\hline CISD & cc-pvdz & 6,9 & 16,0 & 29,2 & 17,4 & 19,4 & 1,11 \\
\hline CISD & aug-cc-pvdz & 10,5 & 19,9 & 32,9 & 21,1 & 19,5 & 0,92 \\
\hline CCSD & cc-pvdz & 7,0 & 16,9 & 29,9 & 17,9 & 19,9 & 1,11 \\
\hline CCSD & aug-cc-pvdz & 11,0 & 21,5 & 34,4 & 22,3 & 20,3 & 0,91 \\
\hline M06HF & aug-cc-pvdz & 11,3 & 20,9 & 34,3 & 22,2 & 19,9 & 0,90 \\
\hline
\end{tabular}


Table S21: Isotropic polarizabilities for functional groups calculated at the DFT/aug-cc-pVDZ level of theory using different functionals. Values in a.u.

\begin{tabular}{|c|c|c|c|c|c|c|}
\hline & & $-\mathrm{NO}_{2}$ & $-\mathrm{NH}_{2}$ & $-S$ & -COOH & $-\mathrm{COO}$ \\
\hline & Type & $\boldsymbol{\alpha}_{\text {iso }}$ & $\boldsymbol{\alpha}_{\text {iso }}$ & $\boldsymbol{\alpha}_{\text {iso }}$ & $\alpha_{\text {iso }}$ & $\boldsymbol{\alpha}_{\text {iso }}$ \\
\hline SVWN & LDA & 26,1 & 14,9 & 26,2 & 22,3 & 24,4 \\
\hline BP86 & GGA & 26,3 & 15,0 & 26,4 & 22,4 & 24,4 \\
\hline BLYP & GGA & 26,6 & 15,1 & 27,0 & 22,7 & 24,5 \\
\hline BPW91 & GGA & 26,1 & 14,9 & 26,1 & 22,2 & 24,2 \\
\hline НСТН & GGA & 26,3 & 15,0 & 26,1 & 22,3 & 24,2 \\
\hline OLYP & GGA & 26,5 & 15,0 & 26,4 & 22,5 & 24,2 \\
\hline BB95 & m-GGA & 26,3 & 15,0 & 26,4 & 22,5 & 24,5 \\
\hline VSXC & m-GGA & 26,3 & 14,8 & 25,9 & 22,1 & 24,1 \\
\hline t-HCTH & m-GGA & 26,4 & 15,0 & 26,3 & 22,2 & 24,3 \\
\hline B3P86 & h-GGA & 25,2 & 14,3 & 25,4 & 21,1 & 23,2 \\
\hline B3PW91 & h-GGA & 25,2 & 14,3 & 25,5 & 21,1 & 23,1 \\
\hline B3LYP & h-GGA & 25,5 & 14,5 & 26,1 & 21,3 & 23,3 \\
\hline O3LYP & h-GGA & 25,8 & 14,7 & 26,0 & 21,7 & 23,5 \\
\hline X3LYP & h-GGA & 25,5 & 14,5 & 26,1 & 21,3 & 23,3 \\
\hline BMK & hm-GGA & 24,1 & 13,8 & 24,6 & 19,6 & 22,1 \\
\hline M06-2X & hm-GGA & 24,3 & 14,0 & 25,5 & 20,2 & 22,3 \\
\hline TPSSh & hm-GGA & 25,8 & 14,7 & 25,8 & 21,7 & 23,7 \\
\hline tHCTHhyb & hm-GGA & 25,7 & 14,5 & 25,9 & 21,5 & 23,5 \\
\hline M06 & hm-GGA & 24,8 & 14,0 & 25,6 & 20,6 & 22,7 \\
\hline M06-HF & hm-GGA & 24,6 & 14,2 & 27,3 & 20,7 & 22,2 \\
\hline M05 & hm-GGA & 25,3 & 14,3 & 26,1 & 21,2 & 22,8 \\
\hline M05-2X & hm-GGA & 24,1 & 13,8 & 25,5 & 20,2 & 22,1 \\
\hline CAM-B3LYP & lc-GGA & 24,8 & 14,1 & 25,9 & 20,9 & 22,7 \\
\hline LC-BLYP & lc-GGA & 24,1 & 13,7 & 25,4 & 20,4 & 21,9 \\
\hline wB97 & lc-GGA & 24,3 & 13,9 & 25,2 & 20,7 & 21,9 \\
\hline LC-OLYP & lc-GGA & 24,0 & 13,7 & 25,2 & 20,3 & 21,8 \\
\hline LC-t-HCTH & lc-GGA & 24,2 & 13,8 & 25,6 & 20,4 & 22,2 \\
\hline LC-HCTH & lc-GGA & 24,2 & 13,8 & 25,6 & 20,4 & 22,0 \\
\hline LC-BB95 & lc-GGA & 23,9 & 13,6 & 25,0 & 19,9 & 21,9 \\
\hline
\end{tabular}




\section{Examples of ill-defined distributed atomic polarizabilities in molecular aggregates}

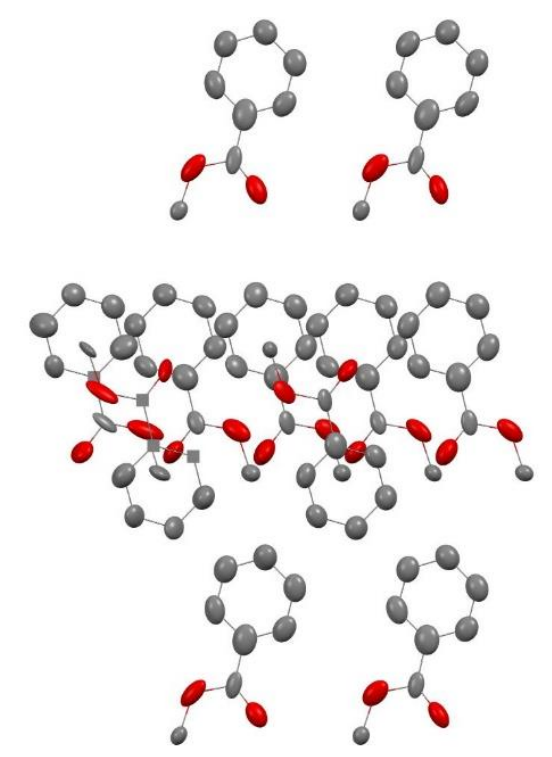

Figure S1: Distributed atomic polarizabilities for the eleven-molecule cluster of methylbenzoate calculated at the M06-HF/aug-cc-pVDZ level of theory. Cubes represent atomic tensors with at least one negative diagonalized component. Hydrogen atoms were hidden for clarity.

Table S22: Diagonalized atomic distributed polarizabilities for the central molecule of the elevenmolecule cluster in methylbenzoate. Values in a.u.

\begin{tabular}{cccccc}
\hline ATOM & $\boldsymbol{\alpha}_{\mathbf{1 1}}$ & $\boldsymbol{\alpha}_{\mathbf{2 2}}$ & $\boldsymbol{\alpha}_{33}$ & $\boldsymbol{\alpha}_{\text {iso }}$ & $\boldsymbol{\Delta} \boldsymbol{\alpha}$ \\
\hline $\mathbf{C 1}$ & 5,9 & 11,8 & 13,1 & 10,2 & 6,7 \\
\hline $\mathbf{C 2}$ & 4,8 & 8,2 & 12,4 & 8,5 & 6,5 \\
\hline $\mathbf{H 3}$ & 0,9 & 1,7 & 4,2 & 2,3 & 2,9 \\
\hline $\mathbf{C 4}$ & 4,6 & 9,0 & 11,6 & 8,4 & 6,1 \\
\hline $\mathbf{H 5}$ & 0,8 & 1,3 & 5,9 & 2,6 & 4,9 \\
\hline $\mathbf{C 6}$ & 4,4 & 10,4 & 12,4 & 9,1 & 7,2 \\
\hline $\mathbf{H 7}$ & 0,8 & 1,4 & 7,1 & 3,1 & 6,0 \\
\hline $\mathbf{C 8}$ & 6,3 & 9,4 & 12,6 & 9,4 & 5,4 \\
\hline $\mathbf{H 9}$ & 1,6 & 3,9 & 5,5 & 3,7 & 3,3 \\
\hline $\mathbf{C 1 0}$ & 3,0 & 9,4 & 11,4 & 7,9 & 7,6 \\
\hline $\mathbf{H 1 1}$ & 0,8 & 4,0 & 7,2 & 4,0 & 5,5 \\
\hline $\mathbf{C 1 2}$ & 1,5 & 5,6 & 10,1 & 5,7 & 7,5 \\
\hline $\mathbf{C 1 3}$ & 4,9 & 5,2 & 7,1 & 5,7 & 2,1 \\
\hline H14 & 1,1 & 1,9 & 5,3 & 2,8 & 3,9 \\
\hline $\mathbf{H 1 5}$ & 1,4 & 2,5 & 4,2 & 2,7 & 2,5 \\
\hline $\mathbf{H 1 6}$ & 1,0 & 1,4 & 4,2 & 2,2 & 3,1 \\
\hline $\mathbf{O 1 7}$ & 5,6 & 10,9 & 16,8 & 11,1 & 9,7 \\
\hline $\mathbf{O 1 8}$ & 3,5 & 7,0 & 16,3 & 8,9 & 11,5 \\
\hline
\end{tabular}


Table S23: Diagonalized atomic distributed polarizabilities for ill-defined atoms in the eleven-molecule cluster of methylbenzoate. Values in a.u.

\begin{tabular}{cccccc}
\hline $\begin{array}{c}\text { ATOM } \\
\text { TYPE }\end{array}$ & $\boldsymbol{\alpha}_{11}$ & $\boldsymbol{\alpha}_{22}$ & $\boldsymbol{\alpha}_{33}$ & $\boldsymbol{\alpha}_{\text {iso }}$ & $\Delta \boldsymbol{\alpha}$ \\
\hline $\mathbf{C}$ & $-47,4$ & 10,4 & 26,7 & $-3,4$ & 67,5 \\
\hline $\mathbf{C}$ & $-6,2$ & 11,4 & 16,3 & 7,2 & 20,5 \\
\hline $\mathbf{C}$ & $-1,8$ & 8,8 & 12,2 & 6,4 & 12,6 \\
\hline $\mathbf{C}$ & $-215,6$ & $-17,4$ & 94,1 & $-46,3$ & 271,7 \\
\hline $\mathbf{H}$ & $-1,7$ & 1,3 & 3,6 & 1,1 & 4,6 \\
\hline $\mathbf{H}$ & $-1,4$ & 1,5 & 4,3 & 1,5 & 5,0 \\
\hline
\end{tabular}

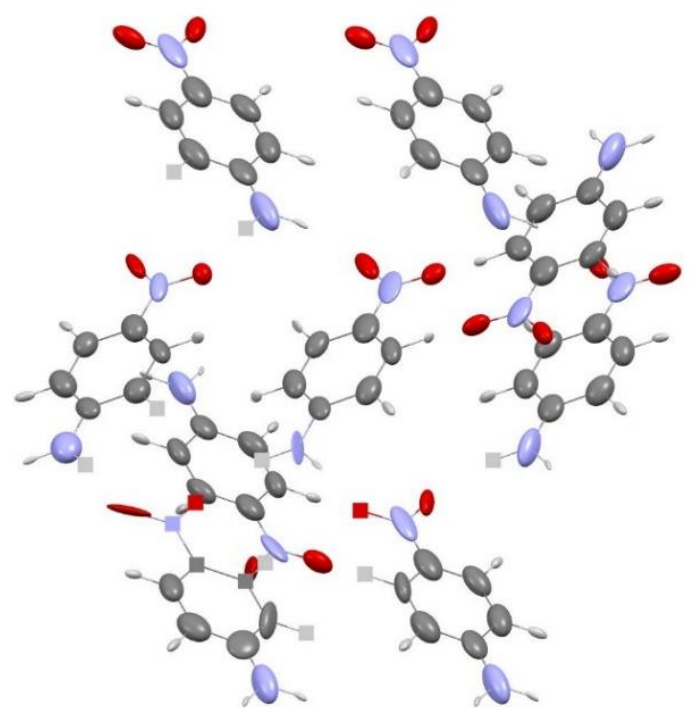

Figure S2: Distributed atomic polarizabilities for the nine-molecule cluster of p-nitroaniline calculated at the M06-HF/aug-cc-pVDZ level of theory. Cubes represent atomic tensors with at least one negative diagonalized component.

\section{Benchmarking of basis and DFT functionals under periodic boundary conditions}

Correlated consisted Dunning basis set (cc-pVnZ) did not converge for $\mathrm{n}$ larger than three for B3LYP or than two for the other tested functionals. aug-cc-pVnZ basis sets were also tested, but convergence was very challenging.

Table S24: Refractive indices for some DFT methods for urea under periodic boundary conditions

\begin{tabular}{cccccc}
\hline Functional & Basis-set & $\mathbf{n}_{\mathbf{a}}$ & $\mathbf{n}_{\mathbf{b}}$ & $\mathbf{n}_{\mathbf{c}}$ & $\mathbf{n}_{\text {iso }}$ \\
\hline B3LYP & cc-pVDZ & 1,38 & 1,38 & 1,66 & 1,47 \\
\hline B3LYP & cc-pVTZ & 1,44 & 1,44 & 1,69 & 1,52 \\
\hline lc-BLYP & cc-pVDZ & 1,34 & 1,34 & 1,59 & 1,42 \\
\hline WB97 & cc-pVDZ & 1,36 & 1,36 & 1,62 & 1,45 \\
\hline cam-B3LYP & cc-pVDZ & 1,36 & 1,36 & 1,63 & 1,45 \\
\hline
\end{tabular}




\section{Electrostatic interaction model (atomic and molecular perturbation) for molecules and functional groups}

Table S25: Diagonalized, isotropic and anisotropic polarizabilities for the central molecule in different clusters of urea, perturbed by atomic dipole moments (ADIM) and molecular total dipole moments (MDIM), located at the center of mass of each peripheric molecule

\begin{tabular}{cccccc}
\hline $\begin{array}{c}\text { CLUSTER } \\
\text { SIZE }\end{array}$ & $\boldsymbol{\alpha}_{11}$ & $\boldsymbol{\alpha} \mathbf{2 2}$ & $\boldsymbol{\alpha} 3 \mathbf{3}$ & $\boldsymbol{\alpha}_{\text {iso }}$ & $\boldsymbol{\alpha}$ \\
\hline \multicolumn{5}{c}{ ADIM } \\
\hline $\mathbf{1}$ & 27,2 & 39,0 & 40,8 & 35,7 & 12,8 \\
\hline $\mathbf{3}$ & 24,2 & 34,8 & 52,3 & 37,1 & 24,6 \\
\hline $\mathbf{5}$ & 21,3 & 37,2 & 52,6 & 37,0 & 27,1 \\
\hline $\mathbf{9}$ & 21,5 & 40,1 & 48,1 & 36,6 & 23,6 \\
\hline $\mathbf{5}$ & 27,2 & 39,0 & 40,8 & 35,7 & 12,8 \\
\hline $\mathbf{1}$ & 24,7 & 34,6 & 51,9 & 37,1 & 23,8 \\
\hline $\mathbf{5}$ & 20,7 & 36,2 & 53,8 & 36,9 & 28,8 \\
\hline $\mathbf{9}$ & 20,0 & 40,4 & 49,0 & 36,5 & 25,8 \\
\hline
\end{tabular}

Table 26: Diagonalized, isotropic and anisotropic polarizabilities for the central molecule in different clusters of succinic acid, perturbed by atomic dipole moments (ADIM) and molecular total dipole moments (MDIM), located at the center of mass of each peripheric molecule

\begin{tabular}{|c|c|c|c|c|c|}
\hline $\begin{array}{c}\text { CLUSTER } \\
\text { SIZE }\end{array}$ & $\alpha_{11}$ & $\alpha_{22}$ & $\alpha 33$ & $\boldsymbol{\alpha}_{\text {iso }}$ & $\Delta \boldsymbol{\alpha}$ \\
\hline \multicolumn{6}{|c|}{ ADIM } \\
\hline 1 & 46,5 & 65,2 & 77,4 & 63,0 & 26,9 \\
\hline 3 & 42,9 & 57,8 & 96,0 & 65,6 & 47,4 \\
\hline 5 & 37,3 & 65,9 & 91,7 & 65,0 & 47,2 \\
\hline 7 & 34,2 & 71,2 & 91,3 & 65,6 & 50,1 \\
\hline 9 & 35,9 & 73,8 & 85,2 & 65,0 & 44,7 \\
\hline 11 & 40,0 & 66,3 & 83,0 & 63,1 & 37,6 \\
\hline \multicolumn{6}{|c|}{ MDIM } \\
\hline 1 & 46,5 & 65,2 & 77,4 & 63,0 & 26,9 \\
\hline 3 & 44,2 & 61,3 & 88,2 & 64,6 & 38,4 \\
\hline 5 & 38,0 & 70,1 & 84,0 & 64,0 & 40,9 \\
\hline 7 & 34,7 & 74,6 & 85,8 & 65,0 & 46,5 \\
\hline 9 & 37,6 & 75,1 & 80,7 & 64,4 & 40,6 \\
\hline 11 & 40,2 & 68,0 & 80,7 & 62,9 & 35,9 \\
\hline
\end{tabular}


Table S27: Diagonalized, isotropic and anisotropic polarizabilities for the central molecule in different clusters of p-nitroaniline, perturbed by atomic dipole moments (ADIM) and molecular total dipole moments (MDIM), located at the center of mass of each peripheric molecule

\begin{tabular}{cccccc}
\hline $\begin{array}{c}\text { CLUSTER } \\
\text { SIZE }\end{array}$ & $\boldsymbol{\alpha}_{\mathbf{1 1}}$ & $\boldsymbol{\alpha}_{\mathbf{2 2}}$ & $\boldsymbol{\alpha}_{\mathbf{3 3}}$ & $\boldsymbol{\alpha}_{\text {iso }}$ & $\boldsymbol{\Delta} \boldsymbol{\alpha}$ \\
\hline $\mathbf{5}$ & \multicolumn{2}{c}{ ADIM } \\
\hline $\mathbf{1}$ & 55,8 & 101,2 & 148,2 & 101,7 & 80,0 \\
\hline $\mathbf{5}$ & 57,1 & 89,1 & 173,2 & 106,5 & 103,9 \\
\hline $\mathbf{7}$ & 54,0 & 94,9 & 185,0 & 111,3 & 116,1 \\
\hline $\mathbf{1}$ & 48,7 & 110,9 & 167,4 & 109,0 & 102,8 \\
\hline $\mathbf{3}$ & 55,8 & 101,2 & 148,2 & 101,7 & 80,0 \\
\hline $\mathbf{5}$ & 56,3 & 92,2 & 171,2 & 106,6 & 101,8 \\
\hline $\mathbf{7}$ & 55,1 & 94,2 & 181,6 & 110,3 & 112,2 \\
\hline
\end{tabular}

Table S28: Diagonalized, isotropic and anisotropic polarizabilities for the central molecule in different clusters of 4-mercaptopyridine, perturbed by atomic dipole moments (ADIM) and molecular total dipole moments (MDIM), located at the center of mass of each peripheric molecule

\begin{tabular}{cccccc}
\hline $\begin{array}{c}\text { CLUSTER } \\
\text { SIZE }\end{array}$ & \multicolumn{1}{c}{$\boldsymbol{\alpha}_{\mathbf{1 1}}$} & $\boldsymbol{\alpha} \mathbf{2 2}$ & $\boldsymbol{\alpha} 3 \mathbf{3}$ & $\boldsymbol{\alpha}$ iso & $\Delta \boldsymbol{\alpha}$ \\
\hline $\mathbf{5}$ & $\mathbf{A D I M}$ \\
\hline $\mathbf{3}$ & 58,7 & 87,0 & 160,8 & 102,2 & 91,3 \\
\hline $\mathbf{5}$ & 54,4 & 80,1 & 185,0 & 106,5 & 119,8 \\
\hline $\mathbf{7}$ & 49,4 & 99,1 & 184,1 & 110,9 & 118,0 \\
\hline $\mathbf{9}$ & 43,0 & 121,6 & 167,4 & 110,7 & 109,0 \\
\hline $\mathbf{1 1}$ & 49,8 & 108,7 & 152,5 & 103,7 & 89,3 \\
\hline $\mathbf{1}$ & 55,2 & 93,6 & 141,3 & 96,7 & 74,7 \\
\hline $\mathbf{3}$ & 58,7 & 87,0 & 160,8 & 102,2 & 91,3 \\
\hline $\mathbf{5}$ & 54,8 & 81,2 & 186,4 & 107,5 & 120,6 \\
\hline $\mathbf{7}$ & 51,8 & 94,5 & 186,9 & 111,0 & 119,6 \\
\hline $\mathbf{9}$ & 46,4 & 118,3 & 173,9 & 112,8 & 110,7 \\
\hline $\mathbf{1 1}$ & 52,0 & 102,3 & 165,0 & 106,4 & 98,1 \\
\hline & 55,5 & 84,7 & 155,8 & 98,7 & 89,4 \\
\hline
\end{tabular}


Table S29: Diagonalized, isotropic and anisotropic polarizabilities for the central molecule in different clusters of methylbenzoate, perturbed by atomic dipole moments (ADIM) and molecular total dipole moments (MDIM), located at the center of mass of each peripheric molecule

\begin{tabular}{cccccc}
\hline $\begin{array}{c}\text { CLUSTER } \\
\text { SIZE }\end{array}$ & $\boldsymbol{\alpha}_{\mathbf{1 1}}$ & $\boldsymbol{\alpha}_{\mathbf{2 2}}$ & $\boldsymbol{\alpha}_{\mathbf{3 3}}$ & $\boldsymbol{\alpha}_{\text {iso }}$ & $\Delta \boldsymbol{\alpha}$ \\
\hline $\mathbf{5}$ & $\mathbf{A D I M}$ \\
\hline $\mathbf{1}$ & 61,4 & 102,2 & 131,4 & 98,3 & 60,9 \\
\hline $\mathbf{3}$ & 56,6 & 98,6 & 152,7 & 102,6 & 83,4 \\
\hline $\mathbf{5}$ & 54,0 & 99,1 & 162,4 & 105,2 & 94,3 \\
\hline $\mathbf{7}$ & 48,5 & 119,4 & 145,4 & 104,4 & 86,9 \\
\hline $\mathbf{9}$ & 58,8 & 106,1 & 124,8 & 96,9 & 58,9 \\
\hline $\mathbf{1}$ & 61,4 & 102,2 & 131,4 & 98,3 & 60,9 \\
\hline $\mathbf{3}$ & 58,3 & 99,6 & 148,0 & 102,0 & 77,8 \\
\hline $\mathbf{7}$ & 56,5 & 98,9 & 156,2 & 103,9 & 86,7 \\
\hline $\mathbf{9}$ & 51,5 & 122,1 & 136,7 & 103,4 & 78,9 \\
\hline & 63,6 & 105,0 & 109,2 & 92,6 & 43,7 \\
\hline
\end{tabular}

Table S30: Diagonalized, isotropic and anisotropic polarizabilities for $-\mathrm{NO}_{2}$ of the central molecule in different clusters of p-nitroaniline, perturbed by atomic dipole moments (ADIM) and molecular total dipole moments (MDIM), located at the center of mass of each peripheric molecule

\begin{tabular}{cccccc}
\hline $\begin{array}{c}\text { CLUSTER } \\
\text { SIZE }\end{array}$ & $\boldsymbol{\alpha}_{11}$ & $\boldsymbol{\alpha} 2 \mathbf{2}$ & $\boldsymbol{\alpha} 33$ & $\boldsymbol{\alpha}$ iso & $\boldsymbol{\Delta}$ \\
\hline \multicolumn{5}{c}{ ADIM } \\
\hline $\mathbf{1}$ & 11,7 & 25,9 & 36,0 & 24,5 & 21,1 \\
\hline $\mathbf{3}$ & 12,3 & 20,7 & 44,3 & 25,7 & 28,7 \\
\hline $\mathbf{5}$ & 11,1 & 24,1 & 46,1 & 27,1 & 30,6 \\
\hline $\mathbf{7}$ & 9,8 & 27,7 & 42,7 & 26,7 & 28,5 \\
\hline $\mathbf{7}$ & $\mathbf{M D I M}$ \\
\hline $\mathbf{3}$ & 11,7 & 25,9 & 36,0 \\
\hline $\mathbf{5}$ & 11,7 & 23,2 & 42,9 & 24,5 & 21,1 \\
\hline $\mathbf{7}$ & 11,0 & 24,0 & 45,0 & 26,0 & 29,7 \\
\hline
\end{tabular}


Table S31: Diagonalized, isotropic and anisotropic polarizabilities for $-\mathrm{NH}_{2}$ of the central molecule in different clusters of p-nitroaniline, perturbed by atomic dipole moments (ADIM) and molecular total dipole moments (MDIM), located at the center of mass of each peripheric molecule

\begin{tabular}{cccccc}
\hline $\begin{array}{c}\text { CLUSTER } \\
\text { SIZE }\end{array}$ & $\boldsymbol{\alpha}_{\mathbf{1 1}}$ & $\boldsymbol{\alpha}_{\mathbf{2 2}}$ & $\boldsymbol{\alpha}_{\mathbf{3 3}}$ & $\boldsymbol{\alpha}_{\text {iso }}$ & $\boldsymbol{\Delta} \boldsymbol{\alpha}$ \\
\hline $\mathbf{5}$ & \multicolumn{2}{c}{ ADIM } \\
\hline $\mathbf{1}$ & 8,4 & 9,4 & 24,8 & 14,2 & 15,9 \\
\hline $\mathbf{3}$ & 6,6 & 9,5 & 31,2 & 15,8 & 23,3 \\
\hline $\mathbf{7}$ & 6,1 & 11,4 & 30,7 & 16,1 & 22,4 \\
\hline $\mathbf{1}$ & 5,9 & 12,4 & 28,3 & 15,5 & 20,0 \\
\hline $\mathbf{3}$ & 8,4 & 9,4 & 24,8 & 14,2 & 15,9 \\
\hline $\mathbf{5}$ & 6,5 & 9,5 & 31,4 & 15,8 & 23,5 \\
\hline $\mathbf{7}$ & 6,5 & 10,3 & 31,7 & 16,2 & 23,5 \\
\hline
\end{tabular}

Table S32: Diagonalized, isotropic and anisotropic polarizabilities for -S of the central molecule in different clusters of 4-mercaptopyridine, perturbed by atomic dipole moments (ADIM) and molecular total dipole moments (MDIM), located at the center of mass of each peripheric molecule

\begin{tabular}{|c|c|c|c|c|c|}
\hline $\begin{array}{c}\text { CLUSTER } \\
\text { SIZE }\end{array}$ & $\alpha_{11}$ & $\alpha_{22}$ & $\alpha_{33}$ & $\boldsymbol{\alpha}_{\text {iso }}$ & $\Delta \boldsymbol{\alpha}$ \\
\hline \multicolumn{6}{|c|}{ ADIM } \\
\hline 1 & 21,2 & 21,9 & 38,7 & 27,3 & 17,2 \\
\hline 3 & 18,0 & 20,4 & 46,2 & 28,2 & 27,1 \\
\hline 5 & 16,7 & 27,6 & 44,1 & 29,5 & 23,9 \\
\hline 7 & 14,3 & 32,9 & 39,2 & 28,8 & 22,4 \\
\hline 9 & 14,6 & 30,3 & 39,4 & 28,1 & 21,7 \\
\hline 11 & 17,1 & 28,0 & 36,6 & 27,2 & 16,9 \\
\hline \multicolumn{6}{|c|}{ MDIM } \\
\hline 1 & 21,2 & 21,9 & 38,7 & 27,3 & 17,2 \\
\hline 3 & 18,2 & 20,1 & 46,8 & 28,3 & 27,7 \\
\hline 5 & 17,8 & 24,8 & 45,5 & 29,4 & 24,9 \\
\hline 7 & 15,7 & 30,9 & 39,8 & 28,8 & 21,1 \\
\hline 9 & 15,4 & 30,8 & 39,7 & 28,6 & 21,3 \\
\hline 11 & 17,2 & 28,7 & 39,0 & 28,3 & 18,9 \\
\hline
\end{tabular}


Table S33: Diagonalized, isotropic and anisotropic polarizabilities for - $\mathrm{COOH}$ of the central molecule in different clusters of succinic acid, perturbed by atomic dipole moments (ADIM) and molecular total dipole moments (MDIM), located at the center of mass of each peripheric molecule

\begin{tabular}{cccccc}
\hline $\begin{array}{c}\text { CLUSTER } \\
\text { SIZE }\end{array}$ & $\boldsymbol{\alpha}_{\mathbf{1 1}}$ & $\boldsymbol{\alpha}_{\mathbf{2 2}}$ & $\boldsymbol{\alpha}_{\mathbf{3 3}}$ & $\boldsymbol{\alpha}_{\text {iso }}$ & $\boldsymbol{\Delta} \boldsymbol{}$ \\
\hline $\mathbf{1}$ & 13,5 & 22,3 & 26,2 & 20,7 & 11,3 \\
\hline $\mathbf{3}$ & 12,2 & 18,7 & 34,3 & 21,8 & 19,7 \\
\hline $\mathbf{5}$ & 10,6 & 21,2 & 33,6 & 21,8 & 19,9 \\
\hline $\mathbf{7}$ & 9,6 & 23,3 & 33,1 & 22,0 & 20,4 \\
\hline $\mathbf{9}$ & 10,1 & 23,5 & 31,2 & 21,6 & 18,5 \\
\hline $\mathbf{1 1}$ & 11,6 & 21,4 & 29,7 & 20,9 & 15,7 \\
\hline $\mathbf{1}$ & 13,5 & 22,3 & 26,2 & 20,7 & 11,3 \\
\hline $\mathbf{3}$ & 12,7 & 20,6 & 30,5 & 21,3 & 15,4 \\
\hline $\mathbf{5}$ & 10,6 & 24,0 & 29,4 & 21,3 & 16,8 \\
\hline $\mathbf{7}$ & 9,5 & 25,4 & 30,3 & 21,7 & 18,8 \\
\hline $\mathbf{9}$ & 10,5 & 26,0 & 27,5 & 21,3 & 16,3 \\
\hline $\mathbf{1 1}$ & 11,5 & 22,8 & 27,1 & 20,5 & 14,0 \\
\hline
\end{tabular}

Table S34: Diagonalized, isotropic and anisotropic polarizabilities for $-\mathrm{COO}^{-}$of the central molecule in different clusters of methylbenzoate, perturbed by atomic dipole moments (ADIM) and molecular total dipole moments (MDIM), located at the center of mass of each peripheric molecule

\begin{tabular}{cccccc}
\hline $\begin{array}{c}\text { CLUSTER } \\
\text { SIZE }\end{array}$ & $\boldsymbol{\alpha}_{\mathbf{1 1}}$ & $\boldsymbol{\alpha}_{\mathbf{2 2}}$ & $\boldsymbol{\alpha}_{\mathbf{3 3}}$ & $\boldsymbol{\alpha}_{\text {iso }}$ & $\Delta \boldsymbol{\alpha}$ \\
\hline $\mathbf{5}$ & $\mathbf{A D I M}$ \\
\hline $\mathbf{1}$ & 11,3 & 20,9 & 34,3 & 22,2 & 20,0 \\
\hline $\mathbf{3}$ & 10,4 & 20,5 & 40,4 & 23,7 & 26,4 \\
\hline $\mathbf{5}$ & 10,1 & 20,2 & 43,2 & 24,5 & 29,4 \\
\hline $\mathbf{9}$ & 9,2 & 24,2 & 38,8 & 24,0 & 25,6 \\
\hline $\mathbf{1}$ & 11,1 & 20,3 & 32,3 & 21,2 & 18,4 \\
\hline $\mathbf{3}$ & 11,3 & 20,9 & 34,3 & 22,2 & 20,0 \\
\hline $\mathbf{5}$ & 10,8 & 20,9 & 37,5 & 23,1 & 23,4 \\
\hline $\mathbf{7}$ & 10,5 & 20,4 & 39,9 & 23,6 & 25,9 \\
\hline $\mathbf{9}$ & 9,7 & 23,8 & 35,6 & 23,0 & 22,5 \\
\hline & 12,3 & 13,4 & 26,0 & 17,2 & 13,2 \\
\hline
\end{tabular}


7. Refractive indices and atomic-dipole interaction model (ADIM) for a 250molecule cluster of urea and succinic acid

Table S35: Refractive indices calculated using the polarizability extracted from the central molecule of urea and succinic acid clusters, perturbed using ADIM. The clusters were built by a $5 \times 5 \times 5$ replication of the unit cells

\begin{tabular}{cccccc}
\hline $\begin{array}{c}\text { CLUSTER } \\
\text { SIZE }\end{array}$ & $\mathbf{n}_{\mathbf{1}}$ & $\mathbf{n}_{\mathbf{2}}$ & $\mathbf{n}_{\mathbf{3}}$ & $\mathbf{n}_{\text {iso }}$ & $\begin{array}{c}\text { Experimental } \\
\text { Cell Volume } \\
(\mathbf{a . u})\end{array}$ \\
\hline $\mathbf{5 0}$ & \multicolumn{6}{c}{ SUCCINIC ACID } \\
\hline $\mathbf{2 5 0}$ & 1,31 & 1,40 & 1,42 & 1,38 & 1683,4 \\
\hline $\mathbf{2 5 0}$ & 1,36 & 1,36 & 1,41 & 1.38 & 980,0 \\
\hline
\end{tabular}

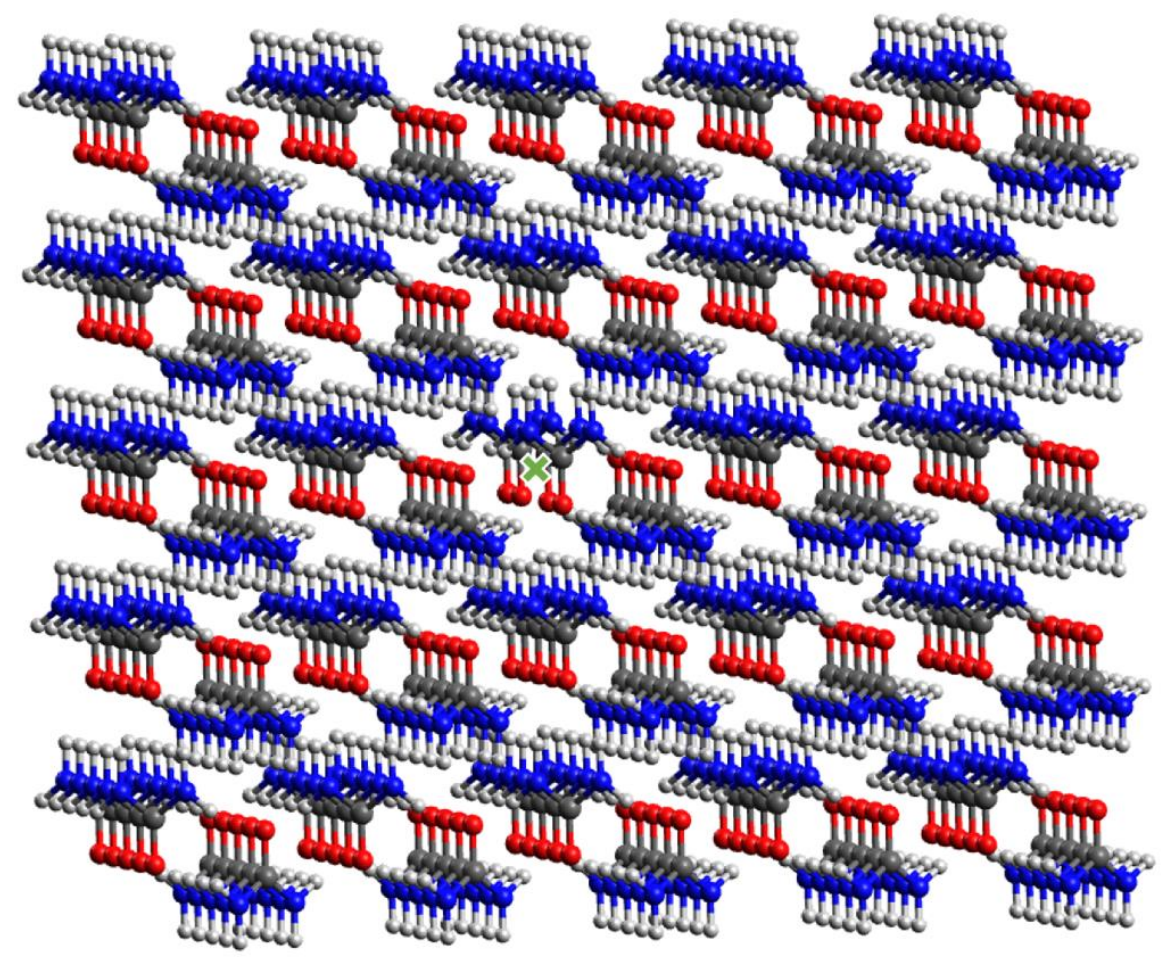

Figure S3: The 250-molecule cluster of urea, showing the central molecule as a green $\mathrm{X}$ 